\title{
РАЗРАБОТКА ФОРМ УПРАВЛЕНИЯ КАЧЕСТВОМ ТРАНСПОРТНЫХ УСЛУГ
}

\begin{abstract}
Определен порядок разработки форм управления качеством транспортных услуг, с учетом особенностей получения информации о ситуации, прогноза ее развития и диагностики, а также анализа результатов на основе коллективной экспертной оценки.
\end{abstract}

\section{1. ВВЕДЕНИЕ}

Процесс управления качеством предоставления транспортных услуг при его, на первый взгляд, простоте при углубленном анализе оказывается достаточно непростым. В нем достаточно много тонкостей и «подводных рифов», которые хорошо знакомы профессиональным менеджерам. В каждом транспортном предприятии (ТП) осуществляется разработка форм управления качеством предоставления транспортных услуг. И в каждом из них практика разработки и реализации таких форм имеет свои особенности, которые определяются характером и спецификой его деятельности, его организационной структурой, действующей системой коммуникаций, внутренней культурой.

Однако есть и общее, характерное для каждого процесса разработки и реализации форм управления качеством предоставления транспортных услуг. Это тот единственный стержень, который формирует технологию разработки и реализации форм управления качеством, используемую в каждом ТП. Ход разработки таких форм управления можно рассматривать как выполнение взаимосвязанного набора этапов и составляющих процесса создания. В каждом конкретном случае этот процесс будет уточнен и индивидуализирован.

\section{2. ПРЕДПОСЫЛКИ РАЗРАБОТКИ И РЕАЛИЗАЦИИ ФОРМ УПРАВЛЕНИЯ КАЧЕСТВОМ ПРЕДОСТАВЛЕНИЯ ТРАНСПОРТНЫХ УСЛУГ}

Одной из отличительных черт теории управления вообще, и в отрасли транспорта в частности, является наличие в ней методов, которые позволяют обрабатывать как количественную, так и качественную (неколичественную) информацию. Возможны различные способы представления процесса разработки и реализации форм управления качеством предоставления транспортных услуг, в основе которых лежат разные подходы к управлению: системный, количественный, ситуационный и др.

На основе структурно-функционального анализа деятельности ТП, а также обобщения литературных источников [2,3], систематизированы этапы процесса

\footnotetext{
${ }^{1}$ Dr Dmytro Ignatenko, Katedra Prawa Transportowego i Logistyki Narodowego Uniwersytetu Transportu w Kijowie, Ukraina.
} 
разработки и реализации форм управления качеством предоставления транспортных услуг, которые представлены на рис. 1. В приведенной укрупненной блок-схеме упорядочены составляющие этого процесса в их технологической последовательности, а также проведено короткое описание особенностей каждой составляющей. Группирование составляющих учитывает роль, которую они играют в процессе управления в ТП.

Подготовка форм управления качеством предоставления транспортных услуг (постановка целей)

Получение информации о ситуации. Прогноз или анализ составляющих процесса). Диагностика ситуации. Выявление проблемной ситуации. Разработка прогноза развития ситуации. Постановка проблемы. Формирование целей.

Разработка форм управления качеством предоставления транспортных услуг

Постановка задания. Генерирование альтернативных вариантов. Отбор основных вариантов решений. Разработка сценариев развития ситуации. Экспертная оценка основных вариантов управляющих действий.

\begin{tabular}{|l|}
\hline \multicolumn{1}{|c|}{ Оценка форм управления качеством, реализация, анализ } \\
результатов \\
Коллективная экспертная оценка. Принятие решения при разработке \\
форм управления качеством предоставления транспортных услуг. \\
Разработка программы (плана) действий. Контроль реализации \\
программы (плана). Анализ результатов развития ситуации. \\
\hline
\end{tabular}

Рис. 1. Основные этапы разработки и реализации форм управления качеством предоставления транспортных услуг

\section{3. ПОДГОТОВКА ФОРМ УПРАВЛЕНИЯ КАЧЕСТВОМ ПРЕДОСТАВЛЕНИЯ ТРАНСПОРТНЫХ УСЛУГ}

Подготовка форм управления качеством предусматривает получение информации о ситуации с использованием современных информационных технологий, в том числе с учетом возможности экспертного оценивания, которые позволяют лицу, принимающему решение (ЛПР), учитывать основные аспекты взаимодействия «ситуация - ЛПР». Это достигается за счет использования качественных и количественных оценок ситуации, формализующихся и не формализующихся, в которой ЛПР осуществляет активные управленческие действия. 
Получаемая информация о реализации форм управления качеством должна быть достоверной и достаточно полной. Недостоверная или недостаточно полная информация может привести к принятию ошибочных и неэффективных решений, которые могут навредить интересам потребителей транспортных услуг. Однако, не меньшие трудности возникают и при наличии избыточной информации, поскольку возникает проблема отбора информации, которая действительно представляет интерес и является важной для своевременного принятия эффективного решения разработки и реализации форм управления качеством предоставления транспортных услуг. Как отмечается [1], руководители транспортных предприятий страдают от избыточной информации, которая не относится к делу. Поэтому в ходе наблюдений важно видеть отличия между релевантной и неуместной информацией и уметь отделять одну от другой.

Важной составляющей при получении и обработке информации о ситуации реализации форм управления качеством является подготовка аналитического материала, который отображает основные особенности и тенденции развития ситуации в ТП. Естественно, что такой аналитический материал должен готовиться менеджерами, которые владеют достаточными знаниями и опытом в сфере, к которой принадлежит ситуация.

Имея необходимую информацию о ситуации и зная цели, к достижению которых стремится ТП, можно переходить к анализу ситуации. Основным заданием анализа ситуации является выявление факторов, которые определяют динамику ее развития. Сначала проводится содержательный анализ и на качественном уровне устанавливаются основные моменты, которые позволяют обнаружить факторы, к изменению измерений и характера действия которых ситуация чувствительна.

Чтобы иметь возможность установить динамику развития ситуации под воздействием тех или иных факторов, необходимо перейти к количественным методам и, таким образом, принять к рассмотрению количественную интерпретацию факторов в виде переменных, значения которых могут изменяться в том или другом диапазоне в зависимости от внешних или внутренних действий ТП.

При анализе ситуации важно выделить ключевые проблемы развития сферы транспортного обслуживания, на которые при целевом управлении процессом необходимо обратить внимание в первую очередь, а также характер их влияния. В этом и заключается задача диагностики ситуации.

На основании проведенного анализа ситуации определяются наиболее чувствительные моменты, которые могут привести к нежелательному развитию событий, фиксируются их признаки. Становятся понятными причины возникновения проблемы. Решение этих проблем необходимо для предотвращения нежелательного развития ситуации в ТП

Достижение поставленных перед ТП целей (если это не только сохранение уровня уже достигнутых результатов), как правило, требует целеустремленных управленческих действий, обеспечивающих развитие ситуации в желаемом направлении.

Особую роль при разработке и реализации форм управления качеством предоставления транспортных услуг играют проблемы, связанные с оценкой развития анализируемых ситуаций и ожидаемых результатов реализации предлагаемых альтернативных вариантов решений. 
При постановке проблемы выделяется и четко описывается еe главное (центральное) звено; определяется круг вопросов, рассмотрение которых необходимо для решения центрального вопроса; находятся содержательные связи и связи по времени, а также соподчинение всего комплекса вопросов, представляющих проблему повышения качества предоставления транспортных услуг. В результате проведения такой работы у ЛПР формируется представление о проблеме и появляется возможность определить пути ее решения.

Диагностика, формулировка и обоснование проблемы - сложное теоретическое и практическое задание. Идентификация проблемы является еще и центральной частью процесса. Если допускается ошибка, то сущность следующих этапов может получить не правильную «наполненность». Да и усилия менеджеров ТП по решению проблемы могут быть направлены на устранение «признаков», а не саму проблему.

Большое значение имеет формирование целей, которые стоят перед ТП и его персоналом относительно решения сформулированной проблемы. Лишь после их выявления можно осуществлять определение факторов, механизмов, закономерностей и ресурсов, которые влияют на развитие ситуации. Формирование целей может осуществляться как непосредственно руководителем ТП, так и коллективно

В настоящее время разработаны методы построения результирующих деревьев целей и деревьев критериев, которые являются результатом коллективной экспертизы для тех случаев, когда точки зрения менеджеров и специалистов, формирующих подходы к управлению качеством предоставления транспортных услуг, могут отличаться [2].

\section{4. РАЗРАБОТКА ФОРМ УПРАВЛЕНИЯ КАЧЕСТВОМ ПРЕДОСТАВЛЕНИЯ ТРАНСПОРТНЫХ УСЛУГ}

Одной из важных составляющих второго блока этапов разработки и реализации форм управления качеством предоставления транспортных услуг является постановка задания. На основании сформированных целей ставится задание разработка мероприятий по реализации целей.

Генерирование альтернативных решений по управлению качеством предоставления транспортных услуг в ТП может осуществляться или непосредственно, или с помощью специальных экспертных процедур. Могут использоваться разные технологии генерирования альтернативных вариантов. Это и технологии на основе метода аналогий, когда разработка форм управления качеством предоставления транспортных услуг основана на использовании опыта решения предыдущих подобных проблем. Это и разные способы синтеза решений из структурированных составляющих, а в более сложных ситуациях - объединение для разработки решения высококвалифицированных менеджеров и специалистов по соответствующим сферам деятельности ТП и др.

После того как разработанные альтернативные варианты форм управления качеством транспортных услуг представлены в виде идей, концепций, возможной технологической последовательности действий, возможных способов реализации предлагаемых вариантов, должен быть осуществлен их предварительный анализ. Он выполняется с целью отсева сознательно нежизнеспособных, неконкурен- 
тоспособных вариантов или вариантов, сознательно дублирующих другие, которые по определенным причинам также предложены для рассмотрения. Технологии отсева могут быть достаточно разными. С этой целью должны привлекаться высокопрофессиональные специалисты, способные отобрать альтернативные варианты форм управления качеством предоставления транспортных услуг, которые достойны последующей, более глубокой проработки и сравнительной оценки.

При отборе основных вариантов форм управления качеством предоставления транспортных услуг необходимо учитывать как их достаточно высокую сравнительную оценку, так и отсутствие дублирования, с тем, чтобы спектр альтернативных вариантов, отобранных для более глубокой проработки, был достаточно полным и в тоже время не избыточным. Должны учитываться также специфические особенности ситуации на рынке транспортных услуг, установленные в процессе его диагностики.

Сценарии ожидаемого развития ситуации играют важную роль при разработке форм управления качеством предоставления транспортных услуг в ТП. Основное задание разработки сценариев - дают ЛПР ключ к пониманию ситуации и наиболее достоверного ее развития. Одним из основных заданий при разработке сценария является определение факторов, которые характеризуют ситуацию и тенденции ее развития в сфере транспортных услуг, а также определение альтернативных вариантов динамики их изменения.

Разработку сценариев развития ситуации целесообразно проводить преимущественно с использованием технологий ситуационного анализа и экспертного оценивания, что дает возможность учитывать как количественную, так и качественную информацию. Следует отметить, что, как правило, приходится рассматривать наиболее достоверные альтернативные варианты ожидаемых изменений ситуации и при наличии управляющих действий, и при их отсутствии.

Анализ нескольких альтернативных вариантов развития ситуации в сфере управления качеством предоставления транспортных услуг обычно оказывается более информативным и способствует выработке более эффективных форм управления качеством. В случае необходимости отобранные ранее основные альтернативные варианты форм управления должны подвергаться адекватной сравнительной оценке и углубленной проработке.

К этому моменту также должна быть сформирована оценочная система, включающая основные факторы (частичные критерии), которые влияют на развитие ситуации по управлению качеством предоставления транспортных услуг, оценку их сравнительной важности, шкалы для определения значений факторов при сравнительной оценке основных альтернативных вариантов управляющих действий в ТП.

Более адекватная оценка альтернативных вариантов управляющих действий в ТП может быть получена при использовании методов коллективного экспертного оценивания.

Конечный результат работы на этом этапе - вынесение суждения о преимуществе альтернатив, предложенных системными аналитиками лицу, которое принимает решение по данной проблеме. На этом процесс разработки форм управления качеством предоставления транспортных услуг заканчивается. 
Следует отметить, что на выбор алгоритма определения результирующей экспертной оценки во многом влияет характер полученной в процессе экспертизы информации, ввиду того, что количественная информация требует одних методов обработки, а качественная - других.

\section{5. ОЦЕНКА ФОРМ УПРАВЛЕНИЯ КАЧЕСТВОМ, ИХ РЕАЛИЗАЦИЯ, АНАЛИЗ РЕЗУЛЬТАТОВ}

При сравнительной оценке альтернативных вариантов форм управления качеством предоставления транспортных услуг могут использоваться специально разработанные оценочные системы, особенно в случае многокритериального оценивания, разработка оценочной системы может также предусматриваться в процессе осуществляемой экспертизы [3]. Коллективная экспертиза является одним из основных инструментов реализации важных форм управления качеством предоставления транспортных услуг в ТП.

Разработка и реализация коллективного оценивания форм управления качеством - одна из наиболее важных процедур процесса организации предоставления транспортных услуг в ТП. В отличие от рассмотренной процедуры определения результирующих экспертных оценок, она предусматривает не только расчет результата коллективной экспертизы, но также: использование специальных методов открытого обсуждения альтернативных вариантов решений; дополнительный обмен информацией между лицами, которые принимают непосредственное участие в процессе принятия решений; согласование противоположных точек зрения; поиск компромисса и другое.

Еще одним важным отличием обсуждаемых коллективных процедур является то, что решения, которые принимаются коллективно, окончательны, тогда как результирующие экспертные оценки служат лишь необходимой базой для предыдущего анализа форм управления качеством предоставления транспортных услуг.

Если разработана конкретная форма управления качеством услуг, то не менее важным заданием является ее успешная реализация. Для этого необходимо составить программу или план действий, поскольку от избранного состава действий, последовательности их осуществления, определенных сроков и, повидимому, самого главного - ресурсов, которые обеспечивают действия исполнителей, которых требует осуществление этих действий, зависит многое. При этом следует отметить, что программа или план - это не раз и навсегда заданная догма. Ведь мы живем и действуем в мире, который изменяется.

Обеспечение эффективной деятельности ТП в сфере управления качеством транспортных услуг предусматривает непрерывный контроль за ходом реализации принятых программ и планов. Современные управленческие технологии, которые используют компьютерное сопровождение, дают возможность одновременно отслеживать ход реализации значительного количества мероприятий в разных сферах и направлениях деятельности ТП.

Реализованный план управленческих действий или его фрагмент, который представляет интерес, должны быть подвергнуты тщательному анализу с целью оценки эффективности реализации форм управления качеством транспортных услуг. Такой анализ должен определить: слабые и сильные места принятых 
решений и планов их реализации; дополнительные возможности и перспективы, которые открываются в результате происходящих изменений; дополнительные риски, которые могут повлиять на достижение намеченных целей.

Эффективный руководитель должен сделать из этого соответствующие выводы и учесть их при разработке и реализации других форм управления качеством предоставления транспортных услуг.

\section{6. ВЫВОДЫ}

Систематизированы особенности процесса разработки и реализации форм управления качеством предоставления транспортных услуг (постановка целей) транспортным предприятием, которые касаются получения информации о ситуации, прогноза или анализа положения (процесса). Предусмотрена диагностика ситуации, выявление ее проблемных аспектов, а также разработка прогноза развития ситуации и на этом основании постановка проблемы и формирование целей управления качеством предоставления транспортных услуг. К блоку этапов разработки форм управления качеством отнесена постановка задания, генерирование альтернативных вариантов и отбор основных вариантов. Обобщены подходы к разработке сценариев развития ситуации и экспертной оценки основных вариантов разработки форм управления качеством транспортных услуг. Составляющие процесса реализации форм управления, анализ его результатов предусматривают коллективную экспертную оценку, принятие окончательного решения, разработку плана действий, контроль реализации плана и анализ результатов развития ситуации на определенном рынке транспортных услуг.

\section{ЛИТЕРАТУРА}

[1] Громов Н.Н., Персианов В.А., Управление на транспорте, Изд.Транспорт, Москва 2008, $336 \mathrm{c}$.

[2] Мишин В. М., Управление качеством: Учебник.- 2-е изд. перераб. и доп, Изд. ЮНИТИ-ДАНА, Москва 2005, 463 с.

[3] Фрейдина Е.В., Исследование систем управления, Изд. «Омега - Л», Москва 2008, 295 с.

\section{DEVELOPMENT OF QUALITY MANAGEMENT FORMS OF TRANSPORT SERVICES}

In the paper it was described the development course of quality management forms of transport services. There were taken into account the features receiving information about a situation, prognosis of its development and diagnostics, as well as analyzed the results on the basis of experts' assessment.

DOI: $10.7862 /$ rz.2012.zim.21 